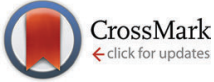

Cite this: Chem. Commun., 2016, 52,6324

Received 9th March 2016, Accepted 4th April 2016

DOI: $10.1039 / c 6 c c 02776 h$

www.rsc.org/chemcomm

\section{Dioxazoles, a new mild nitrene transfer reagent in gold catalysis: highly efficient synthesis of functionalized oxazoles $\dagger$}

\author{
Ming Chen, $\ddagger$ Ning Sun, $\ddagger$ Haoyi Chen and Yuanhong Liu*
}

\begin{abstract}
A gold-catalyzed regioselective [3+2] cycloaddition of ynamides with 1,4,2-dioxazoles was developed and offers a novel approach to obtain highly functionalized oxazoles under mild reaction conditions. 1,4,2-Dioxazole was found to act as an efficient $N$-acyl nitrene equivalent to trigger a facile generation of $\alpha$-imino gold-carbene intermediate through the elimination of a ketone.
\end{abstract}

In recent years, gold-carbene-mediated reactions have attracted considerable attention since they serve as promising intermediates in the synthesis of various types of carbo- or heterocycles. ${ }^{1}$ Compared with $\alpha$-carbonyl gold carbenes, ${ }^{2}$ the generation and reactions of $\alpha$-imino gold carbenes have been less explored. ${ }^{3}$ These highly reactive gold-species are mainly accessed through gold-catalyzed nitrene transfer to alkynes using azides as the nitrene equivalent, as reported by Toste, ${ }^{4 a} \mathrm{Gagosz}^{4 b} \mathrm{Zhang}^{4 c-e}$ and others. ${ }^{4}$ Recently, $2 \mathrm{H}$-azirines, ${ }^{5} \mathrm{~N}$-iminopyridium ylides, ${ }^{6}$ isoxazoles, ${ }^{7}$ benzoisoxazoles $^{8}$ and triazapentalene ${ }^{9}$ have also been used as nitrene equivalents. Despite the impressive progress made so far, the development of new methods for the generation of $\alpha$-imino gold carbenes involving the utilization of less reactive/ sensitive nitrene transfer reagents with high chemo- and regioselectivities under milder reaction conditions is still highly desired. 1,4,2-Dioxazol-5-one a, a cyclic carbonate of hydroxamic acids, and its derivative 1,4,2-dioxazol-5-thione b, were found in 1968 to undergo thermal or photo-induced decomposition leading to highly reactive $N$-acyl nitrene intermediates via the elimination of $\mathrm{CO}_{2}$ or $\mathrm{SO}_{2} \cdot{ }^{10}$ 1,4,2-Dioxazole $\mathrm{c}$ decomposed similarly at elevated temperatures (above $150{ }^{\circ} \mathrm{C}$ ) into isocyanates and ketones. ${ }^{11}$ These attractive and easily accessible heterocyclic compounds are potentially useful as $N$-acyl nitrene precursors in place of hazardous acyl azides, and could produce the $N$-acyl nitrene or

State Key Laboratory of Organometallic Chemistry, Shanghai Institute of Organic Chemistry, Chinese Academy of Sciences, 345 Lingling Road, Shanghai 200032, China.E-mail: yhliu@sioc.ac.cn

$\dagger$ Electronic supplementary information (ESI) available. CCDC 1455247, 1450076 and 1455246. For ESI and crystallographic data in CIF or other electronic format see DOI: $10.1039 / \mathrm{c} 6 \mathrm{cc} 02776 \mathrm{~h}$

\$ These authors contributed equally to this work.
$\mathrm{N}$-acyl nitrenoid intermediates under mild reaction conditions, such as in the presence of a metal catalyst. Recently, Bolm et al. described an elegant light-induced ruthenium-catalyzed synthesis of $\mathrm{N}$-acyl sulfoximines and sulfimides at room temperature via a ruthenium $\mathrm{N}$-acyl nitrene intermediate using dioxazolone a as the nitrene precursor. $^{12}$ More recently, Chang and others ${ }^{13}$ revealed that the substrates a-c could also be used as amidating reagents in metal-catalyzed $\mathrm{C}-\mathrm{H}$ amidation reactions, in which a metal-nitrene complex is proposed to be involved (Scheme 1). During our continuous work on gold-catalyzed oxidative reactions, we hypothesized that these five-membered heterocycles could be employed as a nucleophilic nitrene equivalent to trigger an efficient generation of $\alpha$-imine gold-carbene species through nucleophilic attack of the gold-activated alkyne followed by expulsion of a leaving group. In this design, no metal-nitrene complex is formed, which is different from the other metalcatalyzed reactions shown above. Herein, we describe a novel

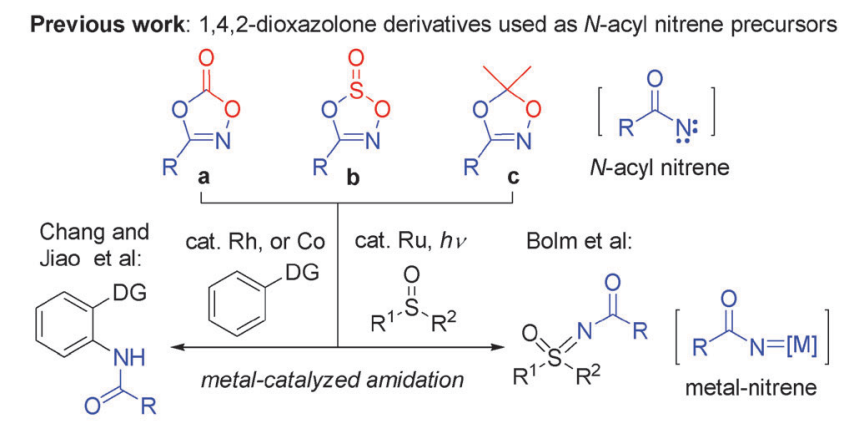

This work: gold-catalyzed nitrene transfer to the alkynes

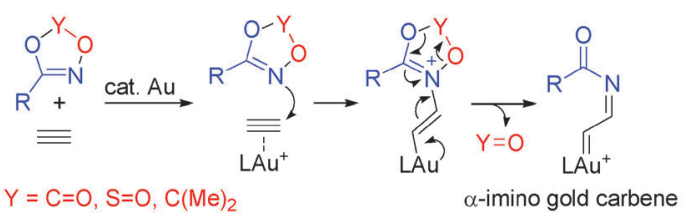

Scheme 1 Metal-catalyzed reactions involving nitrene equivalents of 1,4-2-dioxazolone derivatives and the design of gold-catalyzed nitrene transfer reactions. 
reaction of dioxazole derivatives, which act as a new type of nitrene transfer reagents and undergo gold-catalyzed [3+2] cycloaddition with ynamides, leading to a facile synthesis of highly functionalized oxazoles. ${ }^{6 b-d}$

To test our hypothesis, we initially investigated the reactions of mesylamide-derived ynamide $1 \mathrm{a}$ with three different types of dioxazole derivatives $2 \mathrm{a}-2 \mathrm{c}$ in the presence of $5 \mathrm{~mol} \%$ Johnphos(MeCN)AuSbF 6 (catalyst A) in DCE at room temperature. However, in the case of dioxazolone $\mathbf{2 a}$, a non-clean reaction mixture resulted with significant remaining $2 \mathbf{a}$, possibly as the rapid self-reaction of ynamide had occurred under goldcatalyzed conditions. ${ }^{14}$ No desired cyclization product was observed also in the case of dioxathiazole $\mathbf{2 b}$ (Table 1 , entries 1 and 2). Considering the lower nucleophilicity of $\mathbf{2 a}$ and $\mathbf{2 b}$, we reasoned that employing more nucleophilic dioxazole might be feasible for the successful transformation. Gratifyingly, employing dioxazole $2 \mathrm{c}$ led to the desired 4-amino-oxazole $3 \mathbf{a}$ in a $92 \%$ yield within $2 \mathrm{~h}$ (entry 3). The results implied that an efficient [3+2] cycloaddition of ynamide with dioxazole had taken place, and that the self-reaction of ynamide was mostly suppressed. A similar reaction outcome was found when $N$-heterocyclic carbene gold(I) complex B (IPrAu(MeCN)SbF 6 ) or C $\left(\operatorname{IPrAuNTf}_{2}\right)^{15}$ was used as the catalyst (IPr $=1,3$-bis(2,6-diisopropylphenyl)imidazol-2ylidene) (entries 4 and 5). Various commonly used gold catalysts also catalyze the desired cyclization efficiently, furnishing $\mathbf{3 a}$ in lower yields of $72-87 \%$ (entries $6-8$ ). The reaction could also be performed smoothly in the DCM, THF, toluene or $\mathrm{CH}_{3} \mathrm{CN}$ solvents (entries 9-12). No reaction was observed catalyzed by

Table 1 Optimization of the reaction conditions

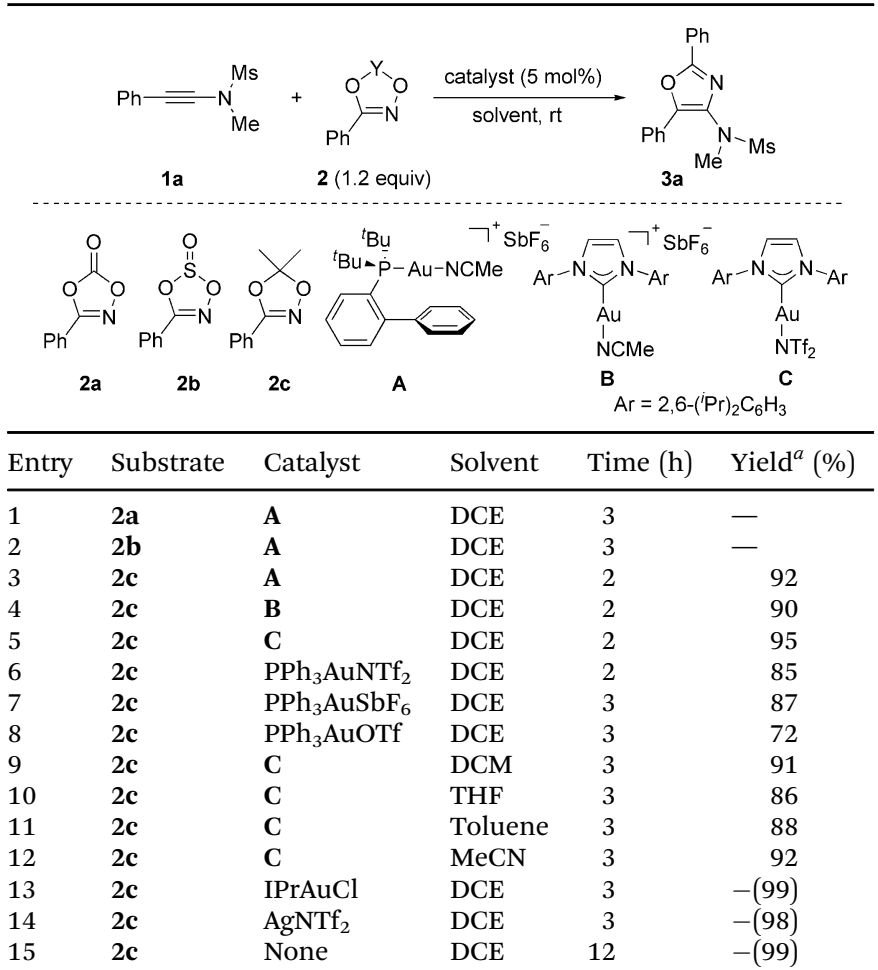

${ }^{a}$ Isolated yields. Ms $=$ methanesulfonyl. The yields of recovered 1a are shown in parentheses.
IPrAuCl or $\operatorname{AgNTf}_{2}$ alone or in the absence of any catalyst (entries 13-15).

Encouraged by these results, we next investigated the substrate scope of the reaction. The scope of ynamides was first investigated using dioxazole $\mathbf{2 c}$ as the reaction partner under the reaction conditions given in Table 1 , entry 5 . The results are shown in Table 2. The effects of the electron-withdrawing groups on nitrogen were first examined. The reactions proceeded very well with tosyl, para-bromobenzenesulfonyl (Bs) and a stronger electron-withdrawing para-nitrobenzenesulfonyl ( $p$-Ns) moiety, furnishing $3 \mathbf{c}-3 \mathbf{e}$ in $72-86 \%$ yields. The more electron-rich ynamide with an oxazolidine group also afforded the corresponding oxazole $3 \mathrm{f}$ in an $89 \%$ yield. $N$-Aryl mesylamide, whenever bearing an electron-neutral, electron-deficient $\mathrm{CF}_{3}$, or electron-rich $\mathrm{MeO}$ substituent on its aromatic ring, was tolerated well in this reaction, leading to $\mathbf{3 b}$ and $\mathbf{3 g}-\mathbf{3 h}$ in $96-99 \%$ yields. $N$-Benzyl mesylamide was also suitable, providing $3 \mathbf{i}$ in an $85 \%$ yield. Next, the effect of the $\mathrm{R}^{1}$ group on the alkyne terminus was examined. For aryl substituted alkynes, a wide range of functionalities, such as $\mathrm{F}, \mathrm{Cl}, \mathrm{CF}_{3}, \mathrm{Me}$ and $\mathrm{MeO}$, on aromatic rings were compatible, furnishing $\mathbf{3} \mathbf{j}-\mathbf{3 n}$ in good to high yields. It was noted that when $p$-MeO-substituted aryl alkyne was used, part of the product precipitated during the reaction process at room temperature, which appeared to interfere with the reaction process.

Table 2 Scope of the ynamides ${ }^{a}$
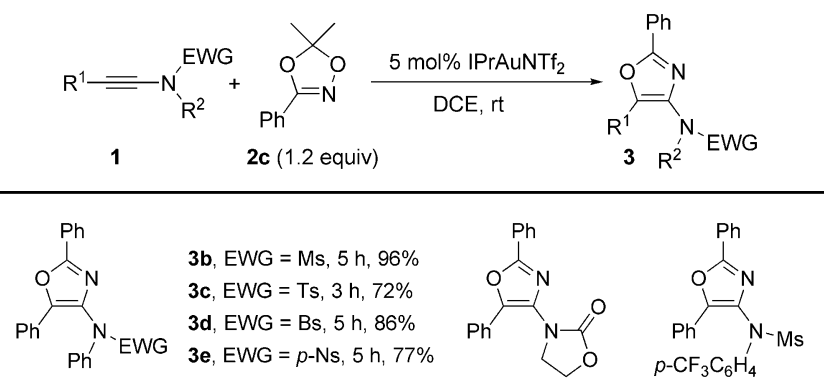

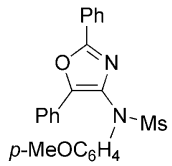

3h, 5 h, $99 \%$
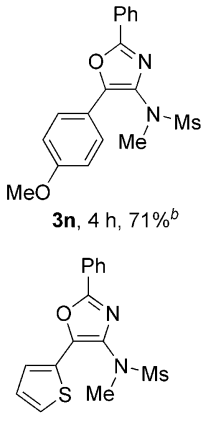

3q, 5 h, $94 \%$

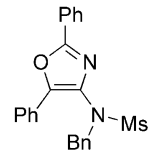

3i, 3 h, $85 \%$ 3f, $4 \mathrm{~h}, 89 \%$

3g, 5 h, $96 \%$<smiles></smiles>

3j, $X=F, 4$ h, $89 \%$ 3k, $\mathrm{X}=\mathrm{Cl}, 3 \mathrm{~h}, 85 \%$ 3I, $X=\mathrm{CF}_{3}, 9 \mathrm{~h}, 83 \%$ $3 \mathrm{~m}, \mathrm{X}=\mathrm{Me}, 3 \mathrm{~h}, 88 \%$
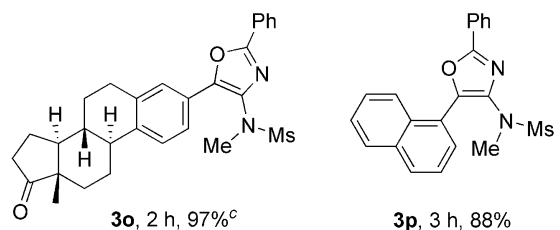

3 p, 3 h, $88 \%$
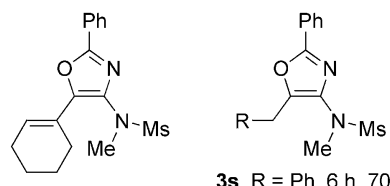

3s, $R=P h, 6 h, 70 \%$ 3t, $R=B u, 5$ h, $30 \%$

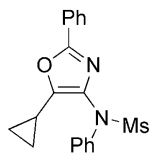

$3 u, 2$ h, $89 \%$

${ }^{a}$ Isolated yields. Ts $=$ toluene-4-sulfonyl, $\mathrm{Bs}=$ para-bromobenzenesulfonyl. $p$-Ns = para-nitrobenzenesulfonyl. ${ }^{b} 50{ }^{\circ} \mathrm{C} .{ }^{c} 80{ }^{\circ} \mathrm{C}$. 
A higher reaction temperature $\left(50{ }^{\circ} \mathrm{C}\right)$ was then required to achieve a better conversion. Ynamide with an 1,3,5(10)-estratrien-3-ol-17one derivative also reacted efficiently to produce the oxazole 30 in a 97\% yield. 1-Naphthyl and 2-thienyl-substituted alkynes converted into the corresponding $\mathbf{3 p}$ and $\mathbf{3 q}$ in excellent yields. Cyclohexenylsubstituted alkyne transformed to the corresponding $3 \mathbf{r}$ in a moderate yield. Alkyl-substituted alkynes, such as benzyl- or cyclopropyl-substituted alkynes, underwent the reaction smoothly to obtain $3 \mathbf{s}$ and $3 \mathbf{u}$ in $70 \%$ and $89 \%$ yields, respectively. However, a pentyl-substituted ynamide afforded $3 \mathbf{t}$ only in a $30 \%$ yield. No alkene product derived from 1,2-C-H insertion of the gold-carbene intermediate was observed in these cases. The results indicated that intramolecular nucleophilic attack of the $\mathrm{N}$-acyl group to gold-carbene is much faster than $1,2-\mathrm{C}-\mathrm{H}$ insertion due to the ease of aromatization.

The scope of dioxazoles was also investigated using ynamide 1a as the reaction partner (Table 3). Due to the lower solubility of the products in DCE, all the reactions were carried out at $80{ }^{\circ} \mathrm{C}$. Under this reaction condition, we were pleased to see that the reactions were quite general with the substituted dioxazoles, since aryl, heteroaryl and alkenyl as well as an alkyl-substituted one were all suitable for this reaction, leading to the highly functionalized oxazoles in good to excellent yields. The reaction efficiency was affected by the nature of aryl substituents: $p-\mathrm{FC}_{6} \mathrm{H}_{4}$ (3v, 90\%), $p-\mathrm{ClC}_{6} \mathrm{H}_{4}$ (3w, 93\%), $p-\mathrm{CF}_{3} \mathrm{C}_{6} \mathrm{H}_{4}$ (3x, 77\%), $p-\mathrm{MeOC}_{6} \mathrm{H}_{4}$ (3y, 92\%). Sterically encumbered $o$-Mesubstituted aryl dioxazole reacted efficiently to afford $\mathbf{3 z}$ in a $90 \%$ yield, suggesting that the steric hindrance had little effect on the reaction course. Heteroaryl-substituted dioxazoles, such as pyridyl, furanyl and a thienyl-substituted dioxazoles, transformed to 3za-3zc successfully in 75-95\% yields. High product yields were also observed in 2-naphthyl or alkenyl-substituted dioxazoles.

Alkyl-substituted dioxazoles, such as methyl, cyclohexyl or even bulky adamantyl, turned out to be also perfect substrates to afford $\mathbf{3 z f - 3 z h}$ in $71-96 \%$ yields. It was noted that in the case of 3zf, dioxazole $2 \mathbf{n}$ was used instead of 3,5,5-trimethyl-1,4,2dioxazole since it is not convenient to prepare the latter with a lower boiling point. Oxazoles constitute important classes of natural products, drugs and biologically active substances. These compounds are commonly prepared by cyclization of an acyclic precursor or ring derivatization. However, the construction of oxazoles through convergent and one-pot methods from readily available substrates is still limited. ${ }^{16}$ Our method provided a mild and efficient route to these compounds.

To demonstrate the practicality of our method, a gram scale reaction was performed. It was found that by using only $2 \mathrm{~mol} \%$ of IPrAuNTf $_{2}$, the reaction of 1 a with dioxazole $2 \mathrm{c}$ at $5 \mathrm{mmol}$ scale delivered oxazole $3 \mathrm{a}$ in a high yield of $89 \%$ (Scheme 2).

The reaction can be extended to other activated alkynes. As shown in Scheme 3, gold-catalyzed reactions of alkynyl ester 4 or alkynyl ketone $\mathbf{5}$ with $\mathbf{2 c}$ afforded the functionalized oxazoles 6 or 7 in $51 \%$ and $50 \%$ yields, respectively. However, when a terminal alkyne, such as phenylacetylene, was used, no clean reaction was observed.

We propose the following reaction mechanism for this novel transformation (Scheme 4). Initially, dioxazole 2 attacks the
Table 3 Scope of the dioxazoles

$$
\text { 1a }
$$
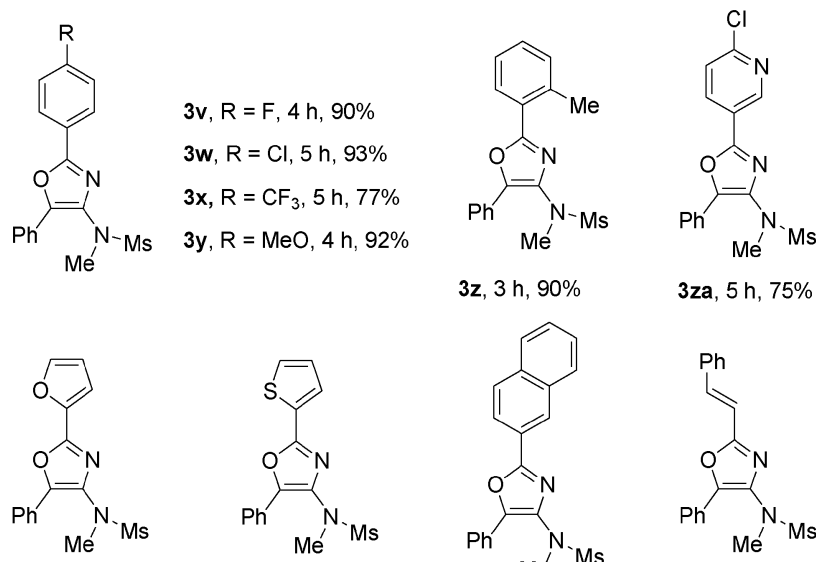

$3 \mathbf{z}, 3 \mathrm{~h}, 90 \%$

3za, 5 h, $75 \%$

$3 z b, 3$ h, $95 \%$

$3 z c, 2$ h, $95 \%$
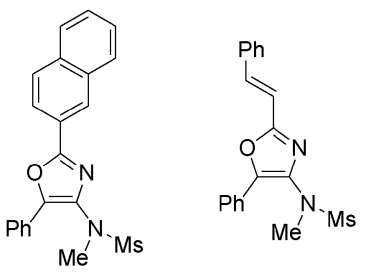

3zd, 2 h, $97 \%$

$3 z e, 2$ h, $97 \%$
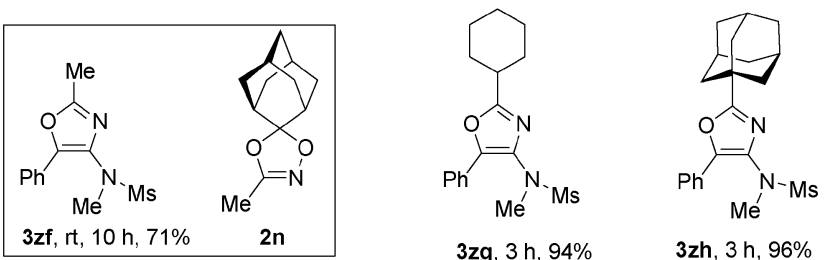

$3 z g, 3$ h, $94 \%$

${ }^{a}$ Isolated yields.

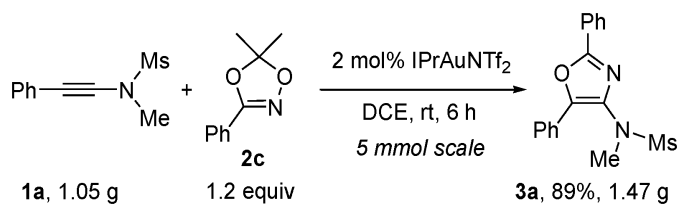

Scheme 2 Gram scale synthesis of $3 a$.

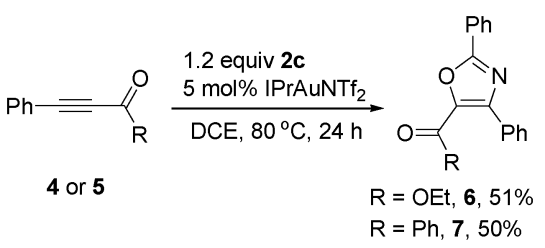

Scheme 3 Reactions of dioxazole with activated alkynes.

gold-coordinated ynamide $\mathbf{8}$ or $\mathbf{8}^{\prime}$ regioselectively at the carbon adjacent to the nitrogen due to the polarity of the ynamide to afford the iminium ion intermediate 10. Subsequently, the ring fragmentation of $\mathbf{1 0}$ generates $\alpha$-imino gold-carbene $\mathbf{1 1}$ with the concomitant elimination of acetone. In fact, acetone was formed quantitatively and could be detected in the crude reaction mixture. ${ }^{17}$ Intermediate $\mathbf{1 1}$ may prefer an $E$-form of 

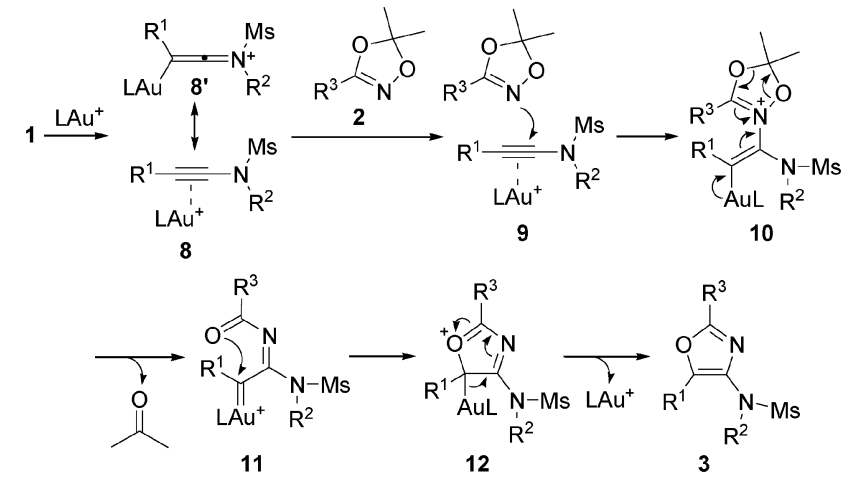

Scheme 4 Possible reaction mechanism.

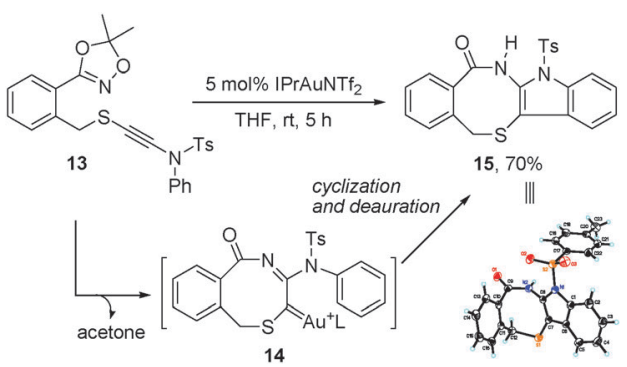

Scheme 5 Trapping of the $\alpha$-imino gold-carbene intermediate.

$\mathrm{C}=\mathrm{N}$ bond due to the steric repulsion with the $\mathrm{R}^{3}$ substituent on dioxazole with the amino moiety, ${ }^{6 a}$ resulting in a cis orientation of an $\mathrm{N}$-acyl group with gold-carbene. Nucleophilic attack of the acyl oxygen in $\mathbf{1 1}$ to gold-carbene ${ }^{18}$ is followed by elimination of the gold catalyst, leading to the oxazole products 3. The reaction pathway involving the formation of $N$-acylaziridine via gold-nitrene followed by cyclization is unlikely, since an oxazole with a different regioselectivity would possibly have resulted. ${ }^{6 b, 19}$

To understand the reaction mechanism, we also tried to trap the $\alpha$-imino gold-carbene intermediate via an intramolecular cyclization of dioxazole-ynamide 13 , since the $\mathrm{C}-\mathrm{O}$ bond formation can be avoided in such a case. To our delight, $\mathbf{1 3}$ cyclized efficiently to give the fused indole derivative $\mathbf{1 5}{ }^{\mathbf{1 5}}$ in a $70 \%$ yield (Scheme 5 ). The results indicated that the $\alpha$-imino gold-carbene $\mathbf{1 4}$ was likely generated in the process, and could be trapped by the $N$-aryl ring, followed by deauration to furnish the cyclized product.

In summary, we disclosed that 1,4,2-dioxazole can be used as an efficient nitrene equivalent in gold-catalyzed nitrene transfer reactions to ynamides. The reaction proceeds under mild reaction conditions to afford highly functionalized oxazoles in good to excellent yields likely via the formation of an $\alpha$-imino gold-carbene intermediate followed by cyclization. This method offers several advantages, such as easily accessible starting materials, high regioselectivity, wide functional group compatibility and high efficiency. Further investigations on the detailed reaction mechanism and application of this chemistry are in progress.

We thank the National Natural Science Foundation of China (Grant No. 21421091, 21372244, 21572256) for financial support.

\section{References}

1 For reviews, see: (a) Gold Carbenes: L. Zhang, in Contemporary Carbene Chemistry, ed. R. A. Moss and M. P. Doyle, Wiley, Hoboken, 2013, p. 526; (b) Y. Wang, M. E. Muratore and A. M. Echavarren, Chem. - Eur. J., 2015, 21, 7332.

2 For reviews, see: (a) J. Xiao and X. Li, Angew. Chem., Int. Ed., 2011, 50, 7226; (b) L. Zhang, Acc. Chem. Res., 2014, 47, 877.

3 For a review, see: P. W. Davies and M. Garzón, Asian J. Org. Chem., 2015, 4, 694 .

4 (a) D. J. Gorin, N. R. Davis and F. D. Toste, J. Am. Chem. Soc., 2005, 127, 11260; (b) A. Wetzel and F. Gagosz, Angew. Chem., Int. Ed., 2011, 50, 7354; (c) B. Lu, Y. Luo, L. Liu, L. Ye, Y. Wang and L. Zhang, Angew. Chem., Int. Ed., 2011, 50, 8358; (d) Y. Xiao and L. Zhang, Org. Lett., 2012, 14, 4662; (e) Z.-Y. Yan, Y. Xiao and L. Zhang, Angew. Chem., Int. Ed., 2012, 51, 8624; $(f)$ Y. Tokimizu, S. Oishi, N. Fujii and H. Ohno, Org. Lett., 2014, 16, 3138; (g) Y. Wu, L. Zhu, Y. Yu, X. Luo and X. Huang, J. Org. Chem., 2015, 80, 11407.

5 (a) A. Prechter, G. Henrion, P. Faudot dit Bel and F. Gagosz, Angew. Chem., Int. Ed., 2014, 53, 4959; (b) L. Zhu, Y. Yu, Z. Mao and X. Huang, Org. Lett., 2015, 17, 30.

6 (a) C. Li and L. Zhang, Org. Lett., 2011, 13, 1738; (b) P. W. Davies, A. Cremonesi and L. Dumitrescu, Angew. Chem., Int. Ed., 2011, 50, 8931; (c) E. Chatzopoulou and P. W. Davies, Chem. Commun., 2013, 49, 8617; (d) A. D. Gillie, R. J. Redd and P. W. Davies, Adv. Synth. Catal., 2016, 358, 226. In the reactions of ref. $6 b-d$, oxazoles were formed via gold-catalyzed [3+2] cycloaddition of pyridine$\mathrm{N}$-aminides with ynamides or alkynyl indoles; $(e)$ H.-H. Hung, Y.-C. Liao and R.-S. Liu, J. Org. Chem., 2013, 78, 7970.

7 A. Zhou, Q. He, C. Shu, Y. Yu, S. Liu, T. Zhao, W. Zhang, X. Lu and L. Ye, Chem. Sci., 2015, 6, 1265.

8 H. Jin, L. Huang, J. Xie, M. Rudolph, F. Rominger and A. S. K. Hashmi, Angew. Chem., Int. Ed., 2016, 55, 794.

9 J. González, J. Santamaría, Á. L. Suárez-Sobrino and A. Ballesterosa, Adv. Synth. Catal., 2016, DOI: 10.1002/adsc.201600022.

10 J. Sauer and K. K. Mayer, Tetrahedron Lett., 1968, 9, 319.

11 H. Nouira, K. Inoue, H. Hattori, T. Okawa and T. Mukaiyama, Bull. Chem. Soc. Jpn., 1967, 40, 664.

12 V. Bizet, L. Buglioni and C. Bolm, Angew. Chem., Int. Ed., 2014, 53, 5639.

13 (a) Y. Park, K. T. Park, J. G. Kim and S. Chang, J. Am. Chem. Soc., 2015, 137, 4534; (b) J. Park and S. Chang, Angew. Chem., Int. Ed., 2015, 54, 14103; (c) Y. Liang, Y. Liang, C. Tang, Y. Yuan and N. Jiao, Chem. - Eur. J., 2015, 21, 16395.

14 S. Kramer, Y. Odabachian, J. Overgaard, M. Rottländer, F. Gagosz and T. Skrydstrup, Angew. Chem., Int. Ed., 2011, 50, 5090.

15 CCDC 1455247 (IPrAuNTf 2 ), 1450076 (3b) and 1455246 (15).

16 For selected papers, see: (a) C. Wan, J. Zhang, S. Wang, J. Fan and Z. Wang, Org. Lett., 2010, 12, 2338; (b) C. Lalli, J. M. Bouma, D. Bonne, G. Masson and J. Zhu, Chem. - Eur. J., 2011, 17, 880; (c) W. He, C. Li and L. Zhang, J. Am. Chem. Soc., 2011, 133, 8482; (d) Y. Luo, K. Ji, Y. Li and L. Zhang, J. Am. Chem. Soc., 2012, 134, 17412; $(e)$ Ref. $6 b-d$. For $\mathrm{Ru} / \mathrm{Cu}$-catalyzed reaction of 1,4,2dioxazol-5-ones with terminal alkynes to oxazoles, see: $(f)$ C. Zhong, B. Tang, P. Yin, Y. Chen and L. He, J. Org. Chem., 2012, 77, 4271. 17 See ESI $\dagger$.

18 (a) S. Kramer and T. Skrydstrup, Angew. Chem., Int. Ed., 2012, 51, 4681; (b) X. Huang, B. Peng, M. Luparia, L. F. R. Gomes, L. F. Veiros and N. Maulide, Angew. Chem., Int. Ed., 2012, 51, 8886.

19 H. Li and R. P. Hsung, Org. Lett., 2009, 11, 4462. 